\title{
THE WISCONSIN VUV/SOFT XRAY FREE ELECTION LASER *
}

\author{
J. Bisognano, R. Bosch, M. Green, H. Hoechst, K. Jacobs, K. Kleman, R. Legg, R. Reininger, \\ R. Wehlitz, UW-Madison/SRC, Madison, Wisconsin \\ J. Chen, W. Graves, F. Kärtner, J. Kim, D. Moncton, MIT, Cambridge, Massachusetts
}

\begin{abstract}
The University of Wisconsin-Madison and its partners are developing a design for a free electron laser (FEL) facility operating in the VUV to soft x-ray range that will be proposed as a new multidisciplinary user facility. Key features of this facility include seeded, fully coherent output with tunable photon energy and polarization over the range 5-900 eV, and simultaneous, independent operation of multiple beamlines. The different beamlines will support a wide range of science from femtochemistry requiring ultrashort pulses with $\mathrm{kHz}$ repetition rates to photoemission spectroscopy requiring high average flux and narrow bandwidth at $\mathrm{MHz}$ rates. The facility will take advantage of the flexibility, stability, and high average pulse rates available from a $\mathrm{CW}$ superconducting linac fed by a photoinjector. This unique facility is expected to enable new science through ultrahigh resolution in the time and frequency domains, as well as coherent imaging and nano-fabrication. This project is being developed through collaboration between the UW Synchrotron Radiation Center and MIT. We present an overview of the facility, including the motivating science, and its laser, accelerator, and experimental systems.
\end{abstract}

\section{CONCEPT}

A Free Electron Laser (FEL) light source is conceived as a new multidisciplinary User facility sited at the University of Wisconsin-Madison. Using high gain harmonic generation laser seeding, the linac-driven FEL source will produce fully coherent, very short (10-30 femtosecond) photon pulses covering the VUV and soft $\mathrm{x}-$ ray range from 5-900 eV. A transform-limited FEL light source of this type does not exist in the US, and its availability would open up a broad range of new research opportunities. The driver will be a superconducting RF linac, which will be operated in a $\mathrm{CW}$ mode and allow pulse repetition formats up to $1 \mathrm{MHz}$ rates or higher. The main requirements for the $2.2 \mathrm{GeV}$ L-Band $\mathrm{CW}$ linac are well within reach of the current state of the art of superconducting RF technology. This technology enables a large number of beamlines, each with flexible pulse format and superb spectral characteristics. The seeded FEL lasing will optimize repeatability, spectral purity, and tunability.

Basic performance goals are:

*Work supported by the University of Wisconsin - Madison, MIT, ONR, AFOSR and DESY. SRC is supported by the U.S. National Science Foundation under Award No. DMR-0537588.

\#JBisognano@src.wisc.edu
- Transform-limited output - longitudinal and transverse

- $\quad$ Pulse power up to $0.1 \mathrm{~mJ}$ Joule

- Many beamlines operating independently and simultaneously

- $\quad$ Complete spectral coverage from 5-900 eV in first harmonic, plus higher harmonics

- Each beamline with wide tuning range

- Peak power and brilliance much larger than current VUV/Soft Xray sources

- Average flux and brilliance much larger than best synchrotrons

- $\quad$ Synchronization of $\sim 10$ fs to user lasers

\section{SCIENCE MOTIVATION}

These powerful, laser-like beams will make the kind of contribution to our understanding of dynamical responses that the current generation of synchrotrons has made to our understanding of static atomic and electronic structures. With full temporal and spatial coherence, with femtosecond timescales, with milli-volt energy bandwidths, and with probe wavelengths down to $1 \mathrm{~nm}$, a new world is opened:

Molecular Dynamics. The combination of high time resolution and spatial coherence of the VUV and soft $\mathrm{x}$ ray FELs allows studies that project out the two-time correlations that lead to key structural fluctuations in chemical reactions. With such a source one can investigate long-range correlations and directly observe the structure-function relationship of systems on the nanometer length scale with femtosecond time resolution. Besides directly imaging molecular scale motions, the FEL properties would also provide unparalleled opportunities to study molecular motors; definition of the molecular origins of reptation in macromolecules; experiments with aligned molecules; achievement of coherent control under both weak and strong field; resolution of structural fluctuations relevant to motions of proteins; and demonstration of solvent dynamics in photodissociation and intermolecular charge transfer.

Atmospheric and Intergalactic Gases. An FEL in the VUV and soft X-ray region will enable new fundamental investigations of the interaction of intense, short-pulsed photon beams with atoms, molecules, clusters and their ions. In particular investigations on ions, which suffer severely from low signal strength due to their low target density, will benefit enormously from an FEL. Also, the high flux and high spectral and spatial resolution of an FEL is needed to accurately model the whole atmosphere and to find the production mechanisms of environmental 
pollution gases. Understanding this particle chemistry has a direct impact on societal concerns such as health. With an FEL light source exciting new possibilities emerge that enable studies of multi-photon processes and of the electronic and structural rearrangement of molecules after photoionization on a femtosecond scale.

Biological Systems. The availability of a tunable VUV and soft x-ray light source allows experiments to reveal the dynamics of biomolecules, their interactions among each other and their response to ionizing radiation. The extreme short-duration of the highly collimated light pulses would revolutionize the real time imaging of biological objects down to the $100 \mathrm{~nm}$ region. Two color femto/picosecond pump probe experiments can track the energy and conformational flow of biomolecules in solution. One can combine two light pulses of different energies, ranging from IR to excite thermal modes in the 100 micron range to soft $\mathrm{x}$-ray energies to access key metal-ligand absorption edges to monitor the energy and conformational flow of in vitro bio-molecules. The FELs also offer the exciting possibility to deliver ionizing radiation of specific energies to cells at high spatial resolution in an effort to disentangle the multitude of different biological pathways. Mapping the response of cells to genomic and component damage would be a major contribution towards understanding the functional units of living organisms.

Time Resolved Imaging and Coherent Scattering. The unprecedented coherence properties of soft x-ray FEL sources will enable unique and incisive probes of nanoscale complexity to be developed. The primary advantage of scattering transversely coherent wave fronts is that this process maps spatial and temporal correlations inside a soft, hard, or biological material into a far-field diffracted wave where they can be easily analyzed. When coupled to atomic, molecular or spin soft x-ray diffractive imaging will allow correlations to be mapped with fewnanometer resolution over a volume the size of a typical cell. Using photon correlation, pump-probe, and probeprobe techniques, fluctuations can be measured over a very broad time scale simultaneously with nanoscale spatial sensitivity.

Exotic Materials, Clusters and Nanostructures. Free electron laser radiation can be used to produce new materials and artificial nanostructures. Novel fabrication techniques based on lithography with coherent light provides a new window of opportunities to directly modify of material properties using femtosecond-scale non-thermal nucleation and growth processes. Currently there exists only limited knowledge on the underlying physics of the relevant microscopic steps in material growth or understanding the structural fluctuations in complex liquids and amorphous solids. These phenomena can be addressed using emerging technologies based on coherent soft x-ray scattering and high- resolution electron spectroscopy.

Condensed Matter and Materials Physics- Resonant Inelastic Xray Scattering (RIXS). The basic purpose of RIXS is to study the elementary excitations in condensed matter, particularly those charge excitations that cannot be accessed by neutron scattering such as plasmons, excitons, orbitons, charge stripes, superconducting gap excitations, etc. However RIXS methods, as currently implemented at storage-ring sources, cannot provide the high (meV) resolution required for studies of such phenomena. There are two new and powerful approaches to RIXS that would be enabled by a soft x-ray FEL. The first is to exploit the huge increase of $10^{6}$ in the timeaverage flux and push existing technology to the limit, i.e. to use multi-stage grating spectrometers, as is used in optical spectrometers, to reject the elastic scattering allowing one to resolve features at much lower energy transfers. The second, which exploits the transverse coherence and time structure of an FEL, is to dispense with grating spectrometers and use photon crosscorrelation techniques to map the phase space area of the pulse, from which the RIXS spectrum can be extracted. With these two methods one has a very high chance of success that would finally permit access to the charge modes that are central to modern condensed matter physics questions.

\section{Condensed Matter and Materials Physics- Ultrahigh}

Resolution ARPES. Angular resolved photoemission spectroscopy, the tool of choice to study quasiparticle behavior and elementary excitations in highly correlated systems such as high Tc superconductors, is nowadays routinely done with energy resolution in the $\mathrm{meV}$ range. The resolving power $\mathrm{E} / \Delta \mathrm{E}$ of modern electrostatic deflection type analyzers has reached its natural limit given by intrinsic inhomogenieties of contact potentials of the analyzer surface resulting in an upper limit of $\mathrm{E} / \Delta \mathrm{E} \sim 4000$. To study complex material properties many experiments would benefit from an increase in the resolving power by at least a factor of ten. This can be realized using time of flight techniques rather than conventional electrostatic deflection type analyzers. While this approach is not suitable for conventional storage rings sources with nanosecond pulse spacing, the FEL pulse pattern with tens-of-femtosecond pulse width enables timing resolution of about 50 picoseconds. With a pulsed FEL source and drift tubes of 3 feet length energy resolution of several $10 \mu \mathrm{eV}$ should be possible.

\section{FEL PERFORMANCE}

The free electron laser beamlines are based on the concept of cascaded harmonic generation with seeding provided by high harmonic generation. Details of the design and modeling appear in the accompanying conference paper of W. Graves et al. [1]. In table 1, two 
sets of representative beamline performance are presented.

Table 1. FEL example performance

\begin{tabular}{|l|l|l|l|l|}
\hline \multicolumn{5}{|c|}{ Beamline 1 at $5.5 \mathrm{eV}$ and Beamline $\mathbf{6}$ at $\mathbf{9 0 0} \mathrm{eV}$} \\
\hline & $5.5 \mathrm{eV}$ & $900 \mathrm{eV}$ & Units \\
\hline Wavelength & 225 & 1.38 & $\mathrm{~nm}$ \\
\hline Beam RMS size & 220 & 34 & $\mu \mathrm{m}$ \\
\hline Beam RMS divergence & 111 & 3.5 & urad \\
\hline Peak power & 4500 & 1400 & $\mathrm{MW}$ \\
\hline Energy/pulse & 135 & 42 & $\mu \mathrm{J}$ \\
\hline Photons/pulse & $1.5 \times 10^{14}$ & $2.9 \times 10^{11}$ & \\
\hline Photons/sec (@1 MHz) & $1.5 \times 10^{20}$ & $2.9 \times 10^{17}$ & \\
\hline Bandwidth & 60 & 60 & $\mathrm{meV}$ \\
\hline & & & \\
\hline Resolution & 0.055 & 9 & $\mathrm{meV}$ \\
\hline Photons/sec (@1 $\left.\mathrm{MHz}, 10^{5} \mathrm{RP}\right)$ & $4 \times 10^{14}$ & $5.1 \times 10^{13}$ & \\
\hline & & & \\
\hline
\end{tabular}

\section{ACCELERATOR AND LASER SYSTEMS}

Accelerator and laser systems are detailed in an accompanying paper [2] together with references. In the following we present a brief summary.

\section{Photo-injector}

The electron injector must supply the continuous stream of electron bunches which have the necessary transverse and longitudinal properties to support the compression system while minimizing the collective effects in the accelerator The bunch profile which best meets these requirement is ellipsoidal with uniform charge density. To create an ellipsoidal bunch with uniform charge density an ultra-short laser pulse with a hemispherical transverse profile can be used which causes a dynamically formed ellipsoidal bunch to be emitted. A possible solution using $200 \mathrm{pC}$ bunches and the FZR 3.6 cell SRF electron gun has been modeled using ASTRA.

\section{Superconducting Linac}

With only 1 milliampere of accelerated beam, several FELs can be simultaneously fed at 1 megahertz repetition frequency each (e.g., for photoemission spectroscopy) with other lines operating at lower repetition rates (e.g., for pump-probe experiments). A linac energy of $2.2 \mathrm{GeV}$ will allow first harmonic operation to $900 \mathrm{eV}$ with a 1.7 $\mathrm{GeV}$ tap-off to feed lower energy lasers effectively. The $1500 \mathrm{MHz}$ CEBAF upgrade cavity [3] provides a reference point, with gradient of $\sim 19 \mathrm{MV} / \mathrm{m}, \mathrm{CW}$ Q of $\sim 8$ $10^{9}$, RF power handling to accelerate $>400$ microamps of beam current, and a dynamic-static heat load of 300 watts for $100 \mathrm{MeV}$ cryomodule.

\section{Bunch Compressors}

A high-quality electron bunch may be created with peak current of $50 \mathrm{~A}$, while the FEL requires a peak current of $1 \mathrm{kA}$ at an energy of $1700 \mathrm{MeV}$. Therefore, we must accelerate the bunch while compressing it by a factor of
20 at sufficiently high energy that space charge effects do not degrade the bunch. We are studying acceleration by an L-band superconducting linac with third harmonic cavities, utilizing two-stage compression of the chirped bunch in magnetic chicanes.

\section{Beam Switchyard}

The desire to run independent beams at repetition rates ranging from hertz to megahertz requires a beam distribution system based on RF separation. The beam separation system consists of a two level binary tree with three separator stages. Four beam ports are possible at each energy. The low energy pickoff point requires another separator with a larger separation angle in order to avoid interference between the high and low energy beamlines. The beams are bent symmetrically giving the minimum bend to each beam for a given separation angle.

\section{Timing Systems}

The gun drive and seeding lasers require synchronization to $10 \mathrm{~s}$ of femtoseconds in order to minimize the electron bunch length required to ensure overlap between the electron bunch and the seed laser pulse. The portion of the pulse which does not interact is thrown away and can represent $50 \%$ or more of the capital costs in the cryogenic, RF and beam dump systems. The RF system and user probe lasers will also need to be synchronized with the gun and seed lasers to comparable precision. In close collaboration between MIT, DESY and FERMI, such a timing distribution system has been developed over the last few years. First deployment and test of this system is under way at the VUV-FEL FLASH at DESY and FERMI at ELETTRA.

\section{ACKNOWLEDGMENTS}

The authors would like to thank the session chairs (John Wright, Paul Evans, Nora Berrah, Stephen Kevan, Robert Austin, David Reis, and Peter Abbamonte) of the New Scientific Opportunities Workshop held in Madison in October 2006 for sharing their insights into the potential for science with VUV/Soft Xray FELs. The authors also would like to thank Brian Sheehy, Juhao $\mathrm{Wu}$, and Paul Emma for very helpful discussions.

\section{REFERENCES}

[1] W. Graves, et al., "Wisconsin XFEL Source Properties: Optimization for Science Applications", these proceedings.

[2] J. Bisognano et al., "A Superconcting Linac Driver for the Wisconsin FEL," these proceedings.

[3] L. Harwood, "Upgrade of the CEBAF Accelerator to $12 \mathrm{GeV}$," Proceedings of the 2006 Linear Accelerator Conference. 